\title{
Buoyant bubbles in a cooling intracluster medium
}

\section{Hydrodynamic bubbles}

\author{
A. Gardini $1^{1,2}$ \\ 1 Department of Astronomy, University of Illinois at Urbana-Champaign, Urbana, IL, 61801, USA \\ e-mail: gardini@astro.uiuc.edu \\ 2 National Center for Supercomputing Applications, Urbana, IL, 61801, USA
}

Received 21 September 2006 / Accepted 9 November 2006

\section{ABSTRACT}

\begin{abstract}
Aims. Over the past several years, numerous examples of X-ray cavities coincident with radio sources have been observed in socalled "cool core" clusters of galaxies. Motivated by these observations, we explore the evolution and the effect of cavities on a cooling intracluster medium (ICM) numerically, adding relevant physics step by step.

Methods. In this paper we present a first set of hydrodynamical, high resolution (1024 effective grid elements), three-dimensional simulations, together with two-dimensional test cases. The simulations follow the evolution of radio cavities, modeled as bubbles filled by relativistic plasma, in the cluster atmosphere, while the ICM is subject to cooling.

Results. We find that the bubble rise retards the development of a cooling flow by inducing motions in the ICM, which repeatedly displace the material in the core. Even bubbles initially set significantly far from the cluster center affect the cooling flow, although much later than the beginning of the simulation. The effect is, however, modest: the cooling time is increased by at most only $25 \%$. As expected, the overall evolution of pure hydrodynamic bubbles is at odds with observations, showing that some additional physics has to be considered to match the data.
\end{abstract}

Key words. galaxies: clusters: general - cooling flows - methods: numerical

\section{Introduction}

Approaching the centers of rich clusters of galaxies, one often finds a remarkable increase in the X-ray luminosity together with a marked decrease in the temperature. Under the conditions present in these clusters, the radiative cooling time of the intracluster medium (ICM) becomes shorter than the cluster lifetime and a "cooling flow" is expected to occur, i.e., a subsonic flow of cooling gas toward the cluster center (see Fabian 1994 for a review). The absence of observations of a reliable repository for the cold gas left some doubts regarding this picture, which were confirmed when a major revision was forced by spectral measurements of the cores of the clusters. Highresolution XMM/Newton (Peterson et al. 2001, 2003; Tamura et al. 2001; Kaastra et al. 2004) spectra and lower resolution ASCA (Makishima et al. 2001) and Chandra (David et al. 2001) spectra show that only a small fraction of the gas is cooling below 1-2 keV, whereas its temperature was expected to be as low as $10^{6} \mathrm{~K}(0.1 \mathrm{keV})$. Hence it is now said that these clusters host a "cool core" instead of a cooling flow, and therefore in the rest of the paper we will refer to them as cool core clusters.

Maintaining gas at $\mathrm{keV}$ temperatures for a period several times longer than its cooling time requires one or more heating mechanisms. Candidate heating mechanisms include thermal conduction by electrons in the ICM (Narayan \& Medvedev 2001; Zakamska \& Narayan 2003; Voigt \& Fabian 2004), reconnection of magnetic fields (Soker \& Sarazin 1990), turbulent mixing (Kim \& Narayan 2003), and the injection of hot plasma by the active galactic nucleus (AGN) hosted by the central galaxy. In this paper we will focus on this last possibility, but we do not exclude the possibility that more than one mechanism is at work.

Recent observations suggest that almost all cool core clusters host radio sources in their centers (Eilek 2004). Often these sources are rather faint, and it is not clear if a correlation exists between their radio power and the strength of the expected cooling flow (Voigt \& Fabian 2004; Kaastra et al. 2004; Bîrzan et al. 2004). However, about $71 \%$ of the $\mathrm{cD}$ galaxies in cool core clusters are radio-loud compared to only $23 \%$ of non-cool core cluster cDs (Burns 1990; Ball et al. 1993), and this suggests a connection between AGN activity and the presence of a cool core, at least in clusters that host a cD.

The shapes of radio sources are determined by the interaction of the jets with their surroundings. The central radio sources in cool core clusters mostly show a disturbed morphology: even when they host a radio-loud core, collimated jets exist only on kpc scales or below. After this the plasma flow continues in a less collimated manner into the ICM, and the lobes take the shape of large plumes or wide tails (see, e.g., Eilek 2004; Jetha et al. 2005). A remarkable exception is Cygnus A, a typical Fanaroff-Riley II (FRII) source, whose jets travel straight for about $100 \mathrm{kpc}$ and terminate in hot spots at the far ends of the lobes (see, e.g., Barthel \& Arnaud 1996).

The interaction of a single jet or a pair of jets with the ICM has been studied extensively both theoretically (Scheuer 1974; Blandford \& Rees 1974; Smith et al. 1983; Begelman \& Cioffi 1989; Heinz et al. 1998; Alexander 2002) and through numerical simulations (see, e.g., Clarke et al. 1997; Rizza et al. 2000; Reynolds et al. 2001, 2002; Krause 2003; Krause \& Camenzind 2003; Zanni et al. 2003, and references therein). These simulations, however, mostly apply only to FRII sources and to the 
phase of AGN activity. This work suggests that jets inflate a cocoon with radio plasma that encompasses both the jets and the AGN, and as the radio source evolves, this cocoon elongates in the jet's direction and assumes a cigar-like shape. The long-term evolution of the radio sources has received less attention; on the one hand, it has been argued that they should continue to expand subsonically while the cocoon remains overpressured with respect to the surrounding medium. On the other hand, it was pointed out that the cocoon evolution would instead be driven by buoyancy when its expansion speed became comparable to the rising buoyancy speed (Churazov et al. 2000). A more detailed picture has been revealed by some recent numerical studies (Reynolds et al. 2002; Basson \& Alexander 2003; Omma et al. 2004; Omma \& Binney 2004; Zanni et al. 2005): after the jets are switched off, they show that lateral instabilities bisect the cocoon in its central region, while its remaining parts start to rise in the cluster atmosphere, due to residual momentum and to buoyancy.

Depressions of the X-ray flux coincident with AGN radio lobes have been observed in cool core clusters and also in some groups and galaxies by ROSAT and Chandra telescopes. They are usually interpreted as cavities in the ICM, produced and filled by the radio-emitting plasma injected by the central AGN as described above. A systematic study of these systems is provided by Bîrzan et al. (2004). Usually, cavities occur in pairs, and the cavities in the same pair are in opposite directions and at about the same distance with respect to the cluster center. In general, they also have similar dimensions, measuring up to some tens of kpc across, and are located some tens of kpc away from the cluster center. However, a pair of huge cavities, roughly $200 \mathrm{kpc}$ in diameter, has been detected by McNamara et al. (2005) in MS0735.6+7421.

Cavities are sometimes surrounded by bright rims of denser and cooler gas (see, e.g., A2052: Blanton et al. 2001), which presumably has been displaced, uplifted, or entrained by the hot plasma during the formation of the cavity or its subsequent motion. In some cases weak shocks are observed surrounding the cavities in clusters (Perseus: Fabian et al. 2006, 2003a; Hydra A: Nulsen et al. 2005b; MS0735.6+7421: McNamara et al. 2005) and in M87 (Forman et al. 2005), but so far strong shocks have been detected only in Centaurus A (Kraft et al. 2003). A weak shock has also been reported around the radio source in Hercules A (Nulsen et al. 2005a), which apparently does not host cavities, and spectral evidence of a shock is reported in A478 (Sanderson et al. 2005). A bow shock in front of radio lobes may have been detected in Cygnus A (Smith et al. 2002; Bałucińska-Church et al. 2005). In the case of a weak shock, it can be assumed that the radio-emitting plasma in the cavities is almost in pressure equilibrium with the surrounding ICM. Thus, due to their low density, the radio lobes will rise by buoyancy in the cluster atmosphere (Gull \& Northover 1973), similarly to the remnants of the cocoon in the models described above.

Chandra X-ray images also show cavities that are not coincident with bright radio lobes, for example in Abell 2597 (McNamara et al. 2001), Perseus (Fabian et al. 2000), and Abell 4059 (Heinz et al. 2002). These structures are referred to as "ghost cavities". The relativistic electrons in the radio lobes are expected to lose enough energy via synchrotron emission to become invisible in the radio after 50 to $100 \mathrm{Myr}$. If this interpretation is correct, the ghost cavities are buoyantly rising relics of a radio outburst that ended at least 50 to $100 \mathrm{Myr}$ ago (Soker et al. 2002).

The commonly observed "cold fronts" discovered by Chandra can also provide hints regarding the effect of AGN on the intracluster medium. Cold fronts are sharp discontinuities in $\mathrm{X}$-ray surface brightness marking boundaries between hotter and colder masses of gas in the ICM. The density and temperature jumps inferred for cold fronts through deprojection analysis are consistent with continuous pressure changes across the fronts, showing that the discontinuities are not shocks (Markevitch et al. 2000). While cold fronts are usually explained as remnants of past merger events, they have also been observed in apparently regular clusters such as RXJ 1720.1+2638 (Mazzotta et al. 2001a), Abell 1795 (Markevitch et al. 2001), 2A 0335+096 (Mazzotta et al. 2003), and MS 1455.0+2232 (Mazzotta et al. 2001b). The gas sloshing induced by the motion of a pre-existing cavity may explain these observations (Mazzotta et al. 2003).

The $\mathrm{H} \alpha$ filaments observed in the core of the Perseus cluster also appear to be related to the upward motion of the cavities. In particular, some filaments seem drawn up in a laminar flow in the wake of a ghost cavity, also tracing well-defined arcs that resemble a circulation flow. This suggests that the medium is not turbulent and has a non-negligible viscosity (Fabian et al. 2003b).

Studies of the heating of the ICM through energy injection by AGN have also been performed using analytical and semianalytical models (Begelman 2001; Ruszkowski \& Begelman 2002; Böhringer et al. 2002; Churazov et al. 2002; Kaiser \& Binney 2003; Brüggen 2003a; Mathews et al. 2003, 2004, 2006; Roychowdhury et al. 2004), which, however, describe these systems in very general terms. This is due to the fact that the dynamics of the hot plasma in the ICM is a very complex problem, owing to its intrinsic three-dimensionality, and to the chaotic interactions between plasma and ICM. While analytical models of buoyant bubbles have been produced (Gull \& Northover 1973; Churazov et al. 2000; Soker et al. 2002; Kaiser et al. 2005), the main tool of investigation consists of numerical simulations. The interaction of the radio sources with the ICM has been simulated so far in different ways. Their formation is often modeled by injecting energy or hot plasma directly into the ICM (Quilis et al. 2001; Brighenti \& Mathews 2002a,b, 2003; Brüggen \& Kaiser 2002; Brüggen et al. 2002, 2005; Brüggen 2003b; Ruszkowski et al. 2004a,b; Dalla Vecchia et al. 2004; Jones \& De Young 2005; Vernaleo \& Reynolds 2006), rather than reproducing detailed AGN jets as discussed above. This approach enables one to study repeated bursts of activity, whereas jet simulations at high resolution are usually unable to cover long evolutionary times. In a different approach, the phase of buoyancy is reproduced by modeling the radio sources as bubbles of hot plasma (Churazov et al. 2001; Brüggen \& Kaiser 2001; Saxton et al. 2001; Robinson et al. 2004; Reynolds et al. 2005), thus neglecting the phase of formation of cavities, and starting from a configuration in pressure equilibrium similar to observations. A review of simulations of hot plasma in clusters is given in Gardini \& Ricker (2004).

We aim to investigate the properties of the ICM, the dynamical evolution of the radio cavities, and their impact on the environment, by means of simulations of increasing complexity and realism. In this paper we show the results of a first set of threedimensional (3D) hydrodynamical simulations in which cavities are modeled as buoyant bubbles in the atmosphere of a cool core cluster, together with two-dimensional (2D) test cases. With respect to previous similar numerical experiments, we achieve a rather high effective numerical resolution $\left(1024^{3}\right.$ grid elements), and account at the same time for the cooling of the gas. Similar simulations have been performed in three dimensions only by Reynolds et al. (2005), who focus on the effect of ICM viscosity on the bubble evolution and therefore neglect cooling. This 


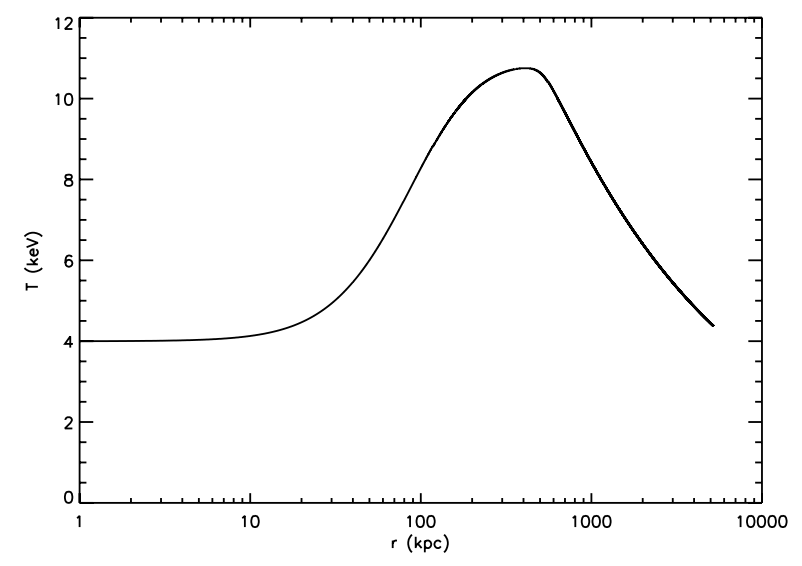

Fig. 1. The temperature profile of the simulated cluster. The temperature rises from about $4 \mathrm{keV}$ at the center up to $11 \mathrm{keV}$ at the scale radius, before decreasing in the outskirts. The profile is derived from the data of Allen et al. (2001) as in Zakamska \& Narayan (2003) for Abell 2390. Beyond the scale radius the profile goes as $T \propto r^{-0.4}$, as suggested by the results of De Grandi \& Molendi (2002).

work deals both with the dynamics of the bubbles and their effect on the cooling of the ICM. We also stress the reliability and robustness of the results, to use them as reference for future simulations.

The paper is organized as follows: in Sect. 2 we describe the characteristics of the simulations and of the code. In Sect. 3 we describe the evolution of the simulations, while the effects of bubbles on the cool core are discussed in Sect. 4. Finally, in Sect. 5 we summarize our results and discuss future developments of this work.

\section{Characteristics of simulations}

We defined the cluster parameters to resemble the observed characteristics of Abell 2390, a massive galaxy cluster with a significant cool core. We model the gravitational potential according to the Navarro et al. (1997) density distribution for dark-matter halos,

$\rho(r)=\rho_{\mathrm{s}}\left[\left(\frac{r}{r_{\mathrm{s}}}\right)\left(1+\frac{r}{r_{\mathrm{s}}}\right)^{2}\right]^{-1}$

and keep it fixed during each run. We set the scale radius $r_{\mathrm{s}}$ to $520 \mathrm{kpc}$, while the scale density $\rho_{\mathrm{s}}=7.07 \times 10^{-26} \mathrm{~g} / \mathrm{cm}^{3}$ is derived by imposing the requirement that the virial mass $\left(M_{200}\right)$ of the cluster be equal to $1.7 \times 10^{15} M_{\odot}$, after assuming the Hubble parameter $h=0.7$ and redshift $z=0$; the corresponding virial radius is then $r_{200} \sim 1.72 h^{-1} \mathrm{Mpc}$.

The initial gas temperature profile is shown in Fig. 1; it is constructed as an analytic adaptation of the profile of Abell 2390 proposed by Zakamska \& Narayan (2003), which in turn reproduces by eye the temperature profile obtained by deprojection from Chandra observations by Allen et al. (2001). The expression for $T(r)$ is

$T(r)=\left(T_{\text {out }}-\left(T_{\text {out }}-T_{\text {in }}\right) \exp \left(-r^{2} /\left(2 \sigma(r)^{2}\right)\right)\right) \times$

$\exp \left(\frac{1}{2}\left(\mu\left(\log (r)-\log \left(r_{\mathrm{s}}\right)\right)-\sqrt{\mu^{2}\left(\log (r)-\log \left(r_{\mathrm{s}}\right)\right)^{2}+0.004}\right)\right)$,

which requires that the temperature rise from $T_{\text {in }}=4 \mathrm{keV}$ at the center up to $T_{\text {out }}=11 \mathrm{keV}$ at the scale radius. Beyond the

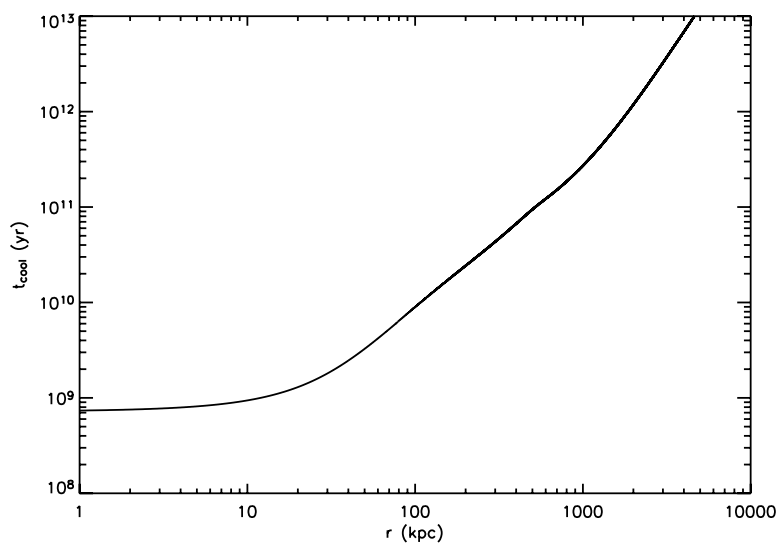

Fig. 2. The value of the cooling time $t_{\text {cool }}=-(\mathrm{d} \ln T / \mathrm{d} t)^{-1}$ as a function of the radius.

scale radius we assume that the gas temperature drops like $T \propto$ $r^{\mu}, \mu=-0.4$, as suggested by the results of De Grandi \& Molendi (2002). The definition of $\sigma$ is

$\sigma(r)=a r+b$

with

$a=\left(\frac{r_{\mathrm{s}}}{3}-\frac{r_{\mathrm{ave}}}{\sqrt{-2 \log 0.5}}\right) /\left(r_{\mathrm{s}}-r_{\mathrm{ave}}\right)$

and

$b=r_{\mathrm{s}}\left(\frac{1}{3}-a\right)$

where the radius $r_{\mathrm{ave}}=80 \mathrm{kpc}$ encompasses the part of the cluster where $T<\left(T_{\text {out }}+T_{\text {in }}\right) / 2$.

The gas density profile is obtained by solving the equation of hydrostatic equilibrium with the condition that the gas fraction inside the virial radius be $f_{b}=0.15$, approaching the cosmic value (see, e.g., Spergel et al. 2003). In Fig. 2 we show the profile of the cooling time $t_{\mathrm{cool}}=-(\mathrm{d} \ln T / \mathrm{d} t)^{-1}$ for the initial conditions according to the cooling function of Sutherland \& Dopita (1993) used in the code. It is worth noting that the region of the cluster where $t_{\text {cool }} \leq 1$ Gyr spans only $r \leq 12 \mathrm{kpc}$. The adiabatic index of the ICM is set to $\gamma=5 / 3$, assuming a monatomic perfect gas. We tested the equilibrium of the initial configuration of the ICM by running a 2D simulation without cooling or bubbles. After $t=500 \mathrm{Myr}$, readjustments in the physical quantities (density, pressure, etc.) amounted at most to $1 \%$.

The rising radio lobes are simulated as a pair of spherical bubbles in the ICM, placed symmetrically with respect to the cluster center. Inside the bubbles, we set the gas density and pressure respectively to $1 / 100$ of and the same value as the ICM's quantities at the same radius; we also impose $\gamma=4 / 3$ in the bubble material because the plasma is relativistic. We did not attempt to include the ICM displaced from these cavities during the phase of bubble formation. In the following we will refer to the two gas components that had $\gamma=5 / 3$ and $\gamma=4 / 3$, respectively, as ICM and bubble plasma.

The different characteristics of the $3 \mathrm{D}$ runs are summarized in Table 1 . The letters CO (cooling only) identify simulations performed without bubbles. In simulations with bubbles, the initials SB (single bubble pair) are followed by the radius $R_{b}=10 \mathrm{kpc}$ of the bubbles and the distance $d_{b}$ of their centers from the center of the cluster. The increasing computational cost did not permit us to run the simulations for their entire cooling 
Table 1. Parameters of the 3D simulations.

\begin{tabular}{crrrrrr}
\hline \hline Name & $L_{\text {box }^{a}}$ & $\Delta x^{b}$ & $R_{b^{c}}$ & $d_{b^{d}}$ & $E_{\text {int }}$ & $t_{\text {final }}$ \\
\hline CO & 4800 & 4688 & - & - & - & 500 \\
CO2 & 600 & 586 & - & - & - & 250 \\
SB1011 & 600 & 586 & 10 & 11 & 337 & 250 \\
SB1020 & 600 & 586 & 10 & 20 & 281 & 250 \\
SB1030 & 600 & 586 & 10 & 30 & 235 & 250 \\
\hline
\end{tabular}

${ }^{a}$ The box size in kpc. ${ }^{b}$ The maximum resolution in pc. ${ }^{c}$ The radius of the bubbles in kpc. ${ }^{d}$ The distance of the center of the bubbles from the center of the cluster in kpc. ${ }^{e}$ The internal energy of the bubbles in units of $10^{57} \mathrm{erg} .{ }^{f}$ The time of the final output of the simulation in Myr.

time, but they cover a significant fraction of it, up to $t=250 \mathrm{Myr}$. A set of simulations with $R_{b}=20 \mathrm{kpc}$ halted at earlier stages of evolution and has not been included in this analysis. All of these simulations are performed on a Cartesian grid. We also created a number of 2D simulations in cylindrical coordinates to test and extend the 3D results. Because of the symmetry of the system, it can be noted that the same amount of information extracted by a 3D simulation could be obtained through a faster and computationally cheaper 2D counterpart. We noticed, however, that the two bubbles in these 2D runs evolve in a similar but not fully symmetric manner. This is probably due to a greater amplification of rounding errors in this particular system of coordinates with respect to the Cartesian grid. The evolution of the bubbles and their effect on the medium are nonetheless in agreement with the $3 \mathrm{D}$ cases until they are available, and this encouraged us to trust the $2 \mathrm{D}$ results, at least as checks or hints of the overall evolution of the system. We will not discuss the 2D runs extensively, but we will report their results when necessary.

The 3D computational box is centered on the cluster center and in general spans $600 \mathrm{kpc}$; its dimensions are a good compromise between resolution and the need to avoid spurious boundary effects. Because we chose reflecting boundary conditions to conserve mass and energy, the box size has to be large enough that the cooling flow and the bubbles are unaffected. We achieve a maximum resolution of $\sim 586 \mathrm{pc}$, comparable to the electron mean free path in the cluster core, when magnetic fields are neglected (Sarazin 1988). The CO model, which uses a box size of $4800 \mathrm{kpc}$ and a resolution of $\sim 4.7 \mathrm{kpc}$, is a test case for the development of the cooling flow in a larger box. The cooling function of Sutherland \& Dopita (1993) is implemented in the code; we shut off cooling below a temperature of $10^{4} \mathrm{~K}$ and assume an ICM metallicity of $\sim 0.3 Z_{\odot}$.

All simulations are performed using FLASH (Fryxell et al. 2000), a parallel Eulerian hydrodynamic code that implements the piecewise-parabolic method (PPM) of Colella \& Woodward (1984) on an adaptive mesh. Grid cells are grouped in blocks, which are refined or derefined according to the maximum value of the second derivative of pressure or density (Löhner 1987). In 3D simulations the volume is divided into $4^{3}$ blocks at the coarsest level of refinement, and each block contains $8^{3}$ cells. Up to 6 levels of refinements are allowed, with a factor of two refinement between levels.

FLASH manages mixtures of gases using the method of Colella \& Glaz (1985) to handle general equations of state. For the purposes of this work it is worth noting that each fluid element in the simulation is composed of a fraction of both the ICM and the bubble plasma, and that all of the physical quantities, such as density, temperature, and pressure, are evaluated on

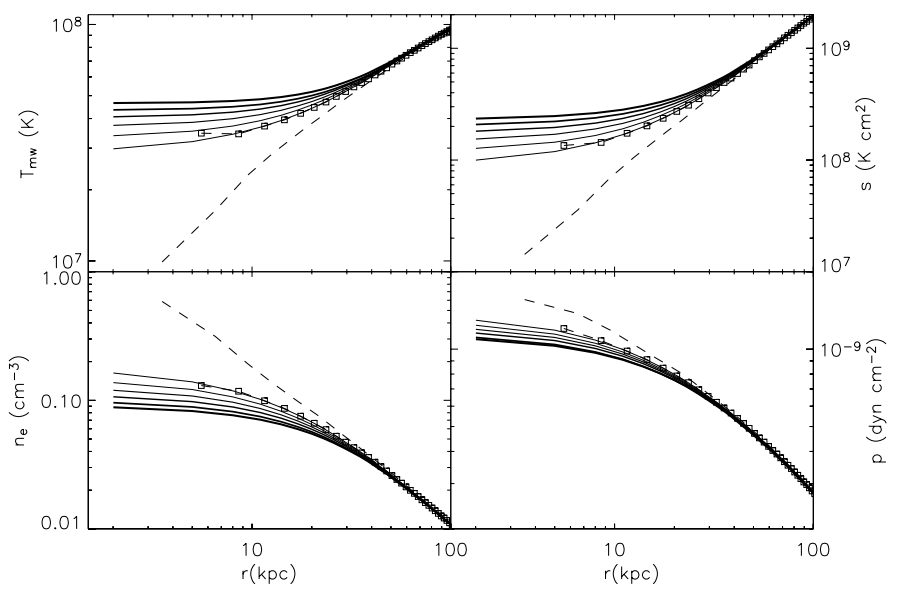

Fig. 3. The evolution of the profiles of some of the ICM quantities due to cooling, in the absence of cavities. The plot shows the evolution of temperature $T$, electron density $n$, entropy $s=T / n^{2 / 3}$, and pressure $P$ in the central part of the cluster. Profiles are obtained from the $\mathrm{CO} 2$ (solid lines) and CO (dashed lines) simulations by averaging over radial bins, assuming a bin interval of $3 \mathrm{kpc}$. The time evolution for the CO2 simulation is represented by the line thickness, which decreases with time. Profiles are computed with an interval of $50 \mathrm{Myr}$ from the initial conditions up to $t=250 \mathrm{Myr}$. The dashed lines represent the profiles of the same quantities in the CO simulation at $t=250 \mathrm{Myr}$ and $t=$ 500 Myr. Squares have been added to the profiles of the CO simulation at $t=250 \mathrm{Myr}$ to aid comparison with the $\mathrm{CO} 2$ case.

the mixture of the two species. In particular, a weighted average adiabatic index $\gamma$ is assigned to each cell as

$\frac{1}{(\gamma-1)}=\sum_{i} \frac{X_{i}}{\left(\gamma_{i}-1\right)}$

where $X_{i}$ is the mass fraction of the $i$ th species and $\gamma_{i}$ its adiabatic index. This left us with two possible problems: on the one hand, mixing is not properly treated in these simulations because the diffusion of species is not explicitly accounted for. On the other hand, the temperature in the plasma elements drops quickly as they are polluted by the ICM, but the relativistic value $\gamma=4 / 3$ of their adiabatic index does not change. We address the possible effects of these inconsistencies in the following sections.

\section{Evolution of simulations}

\subsection{The development of the cooling flow}

The simulations named $\mathrm{CO}$ and $\mathrm{CO} 2$ in Table 1 allow the ICM to cool, in the absence of bubbles or any source of heating. They reproduce the effect of cooling on the ICM starting from the equilibrium configuration assumed in the initial conditions. The simulations are identical except for the box size and the corresponding resolution.

Figure 3 shows the evolution of temperature $T$, electron density $n$, entropy $s=T / n^{2 / 3}$, and pressure $P$ in the central part of the cluster due to cooling. Profiles are obtained for the $\mathrm{CO} 2$ and $\mathrm{CO}$ simulations by averaging on density and assuming a bin interval of $3 \mathrm{kpc}$. The temporal evolution for the $\mathrm{CO} 2$ simulation is represented by the line thickness, which decreases with time: profiles are computed at intervals of $50 \mathrm{Myr}$ from the initial conditions up to $t=250 \mathrm{Myr}$. The dashed lines represent the profiles of the same quantities in the CO simulation to $t=250 \mathrm{Myr}$ and $t=500 \mathrm{Myr}$; at $t=250 \mathrm{Myr}$ we also differentiate the symbols to facilitate visual comparison. The figure shows that the profiles at 


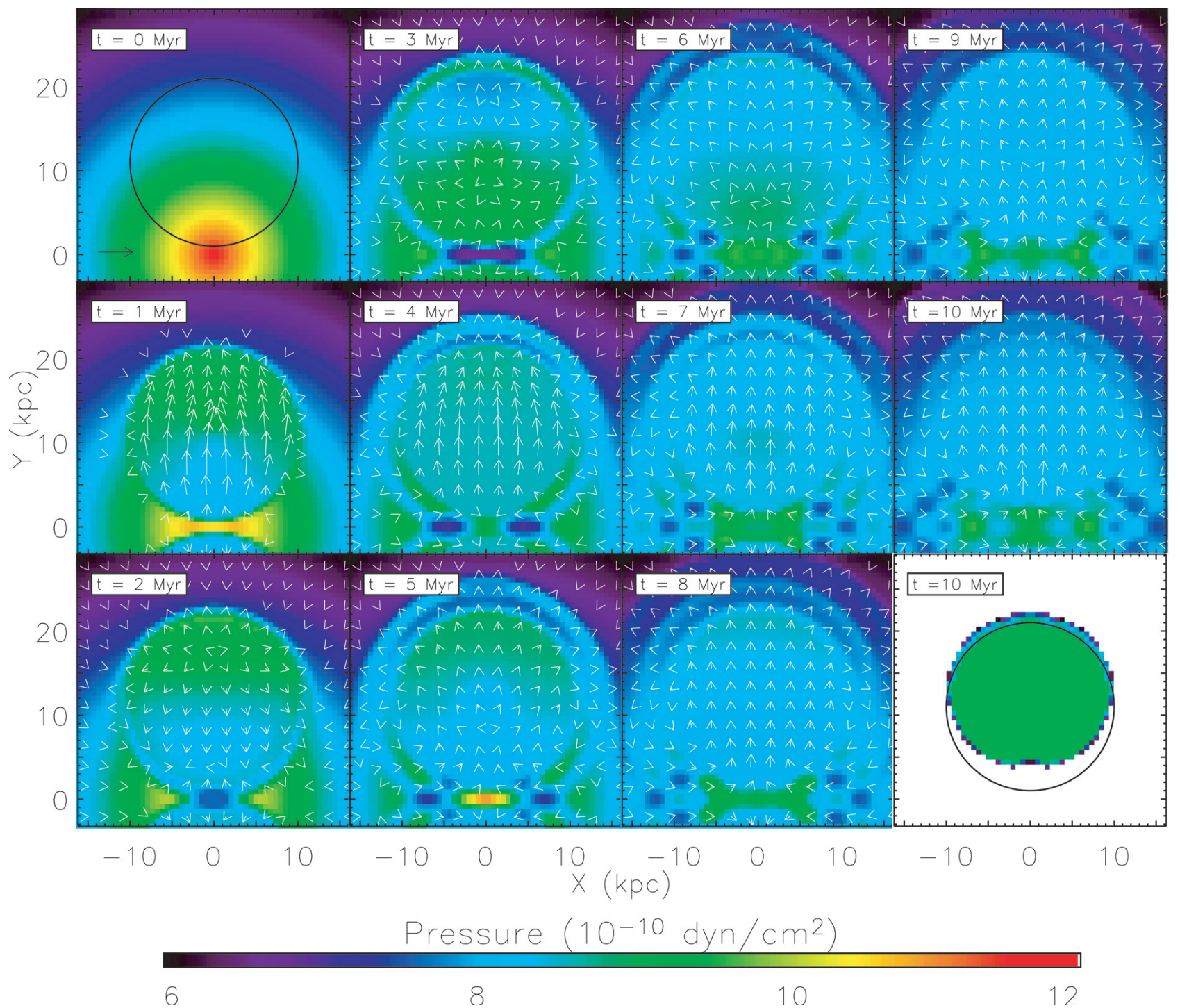

Fig. 4. The evolution of the pressure field from $t=0$ up to $t=10 \mathrm{Myr}$ for the simulation SB1011 in the region around the bubble, with the velocity field superimposed. The first picture shows the border of the bubble as well as a velocity vector corresponding to $v=1000 \mathrm{~km} \mathrm{~s}{ }^{-1}$. The last snapshot shows the region occupied by the fluid elements containing at least $90 \%$ hot plasma at $t=10$ Myr, with the initial position of the bubble superimposed on it. The bubble rises $\sim 2 \mathrm{kpc}$ in the first $10 \mathrm{Myr}$.

$t=250 \mathrm{Myr}$ practically coincide, to the limit of resolution; this implies that the finite size of the box does not induce spurious effects in the $\mathrm{CO} 2$ simulation.

As expected, these runs show the establishment of a homogeneous cooling flow that evolves in a runaway manner (White \& Sarazin 1987). Because of the long cooling time, it is not surprising that the temperature of the core drops only to $T=3 \times 10^{7} \mathrm{~K}$ after the first $250 \mathrm{Myr}$. Using a 2D simulation with the same resolution as the $\mathrm{CO} 2$ case, we checked that the central temperature falls to the minimum value between $t=390$ and 400 Myr. We will use the profiles obtained in the $\mathrm{CO} 2$ case as references for the same quantities computed in the presence of bubbles.

\subsection{The rise of the bubbles}

The rise of spherical bubbles of plasma in a cluster atmosphere has been described in many papers (e.g., Churazov et al. 2000). We observe that the evolution of bubbles in the ICM quite closely resembles the dynamics of powerful explosions in the earth's atmosphere, or more generally of "thermals", which are spherical bubbles of hot air in pressure equilibrium with the surrounding colder air. In the latter case, the main difference resides in the fact that thermals are only slightly hotter and less dense than the surrounding air; this produces a rather different behavior, and thermals are able to rise in the atmosphere much higher than their initial diameter, expanding linearly with time (Turner 1973). Studies of thermals can hence adopt the Boussinesq approximation in computations and perform experiments using liquids. Nevertheless, some of the behavior of thermals, such as their internal motions, presents remarkable similarities to the behavior of plasma bubbles. The behavior of spherical bubbles in water also resembles, for a proper choice of parameters, the behavior of plasma bubbles (e.g., Walters \& Davidson 1963).

The evolution of the $3 \mathrm{D}$ simulations with bubbles is shown in Figs. 4 to 9 through maps constructed by cutting the simulations along one of the planes of symmetry that contain the centers of the cluster and the bubbles. We concentrate on the simulation SB1011 as representative of the sample, and will refer to the other 3D simulations only when their behavior is quite different with respect to it. 


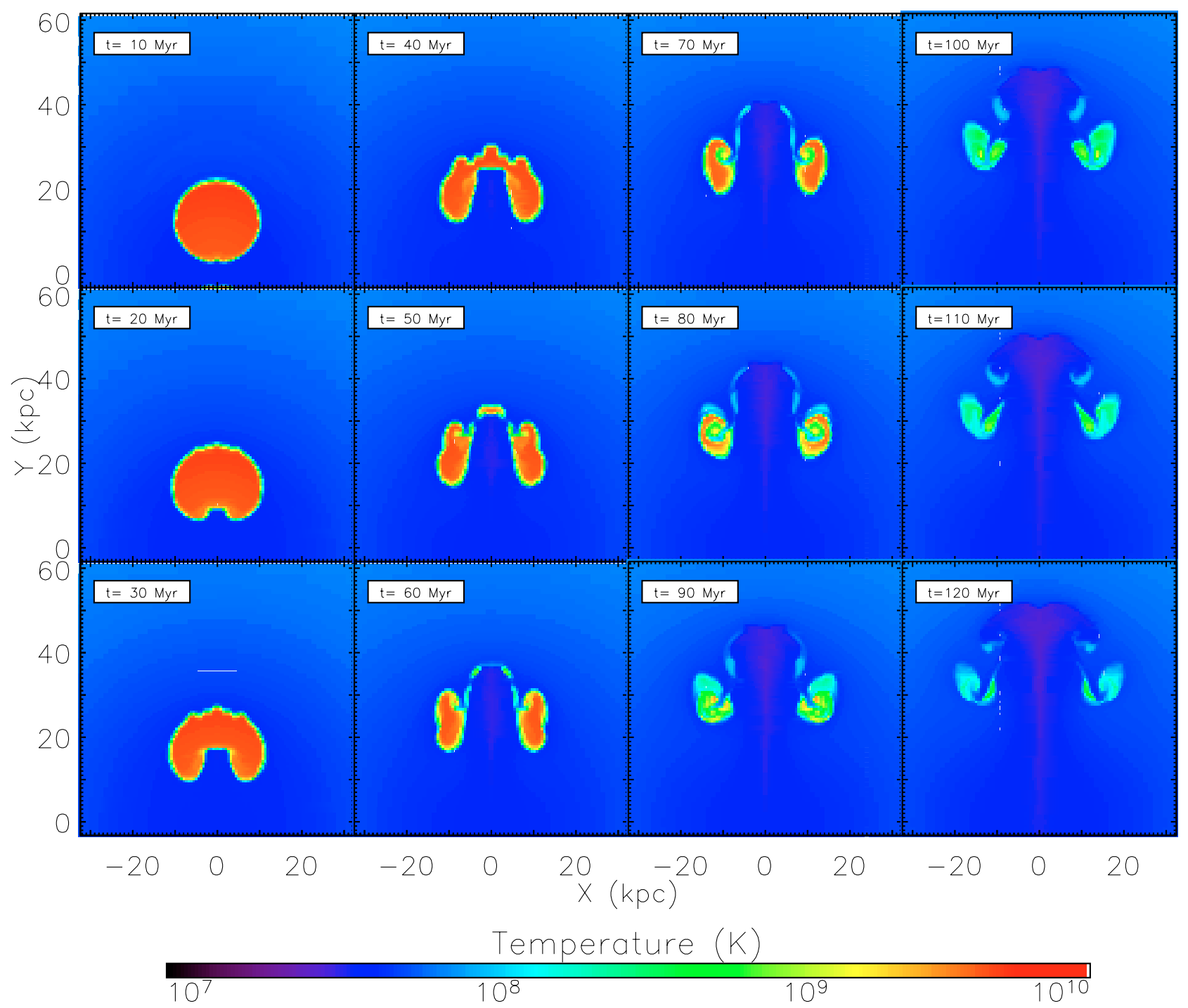

Fig. 5. Map of the temperature in the SB1011 simulation showing the evolution of the plasma bubble in the first 120 Myr. The pictures clearly show the effect of the entrained cold gas, which penetrates and finally bisects the ICM bubble, leaving a vortex ring.

We paid some attention to the first stages of the evolution, namely the starting of the bubble from rest and the generation of sound waves. This phase is usually neglected in the literature as transient and unrealistic (see, however, Churazov et al. 2002), despite the fact that sound waves are often clearly visible in figures. In Fig. 4 we show the evolution of the pressure and velocity fields during the first $10 \mathrm{Myr}$. The plots show how the bubble plasma oscillates vertically and generates sound waves, while motions are driven into the adjacent ICM at the same time. At the time of the last snapshot, a vortex circulation directed upward along the bubble axis and downward on the bubble surface (Hill 1894; Batchelor 1967) is established in the plasma and in the surrounding ICM. This kind of circulation enables the bubble to rise steadily (Turner 1973).

During the rise that follows the initial transient phenomena, each bubble is affected by instabilities on its surface due to lack of surface tension. In particular it will suffer RayleighTaylor (RT) instabilities on the top and Kelvin-Helmoltz (KH) instabilities on its flanks. In the end, however, it is the vortex circulation itself that mostly affects the bubble. Indeed, in an ideal case a stagnation layer should be established in the ICM below the bubble, but in reality, as the bubble rises, the fall of pressure at its bottom draws the ICM upward, where it is entrained in the vortex circulation, thus acting as an effective RT instability.

The evolution of the rising bubbles is depicted in Fig. 5 with intervals of $10 \mathrm{Myr}$ up to $t=120 \mathrm{Myr}$ and in Figs. 6 to 8 with intervals of $50 \mathrm{Myr}$ up to $t=250 \mathrm{Myr}$. As long as the bubble rises, a trunk of denser material is entrained in its wake, which penetrates it from below and gives it a mushroom shape. As expected in the development of RT instabilies, the uplifted material rises faster and eventually bisects the bubble, which then assumes the shape of a rotating torus (or vortex ring). It is worth noting that the ICM entrained by the bubble cools by expansion because of the lower pressure, and at some times it is the coldest material in the cluster. As the evolution goes on, the lower part of the trunk falls back to the cluster center, while the upper part remains at large radii as long as it is driven in the vortex circulation.

Defining the borders of the bubbles becomes a harder task as the simulation proceeds. Figure 8 shows the fraction of the bubble plasma in the fluid elements. Inspection of these values shows clearly that all of the hot plasma has mixed completely with the ICM after $100 \mathrm{Myr}$. In particular, the plasma picked up along the bubble axis by the rising ICM cools rapidly to low temperature due to the high density of the entrained ICM and should 


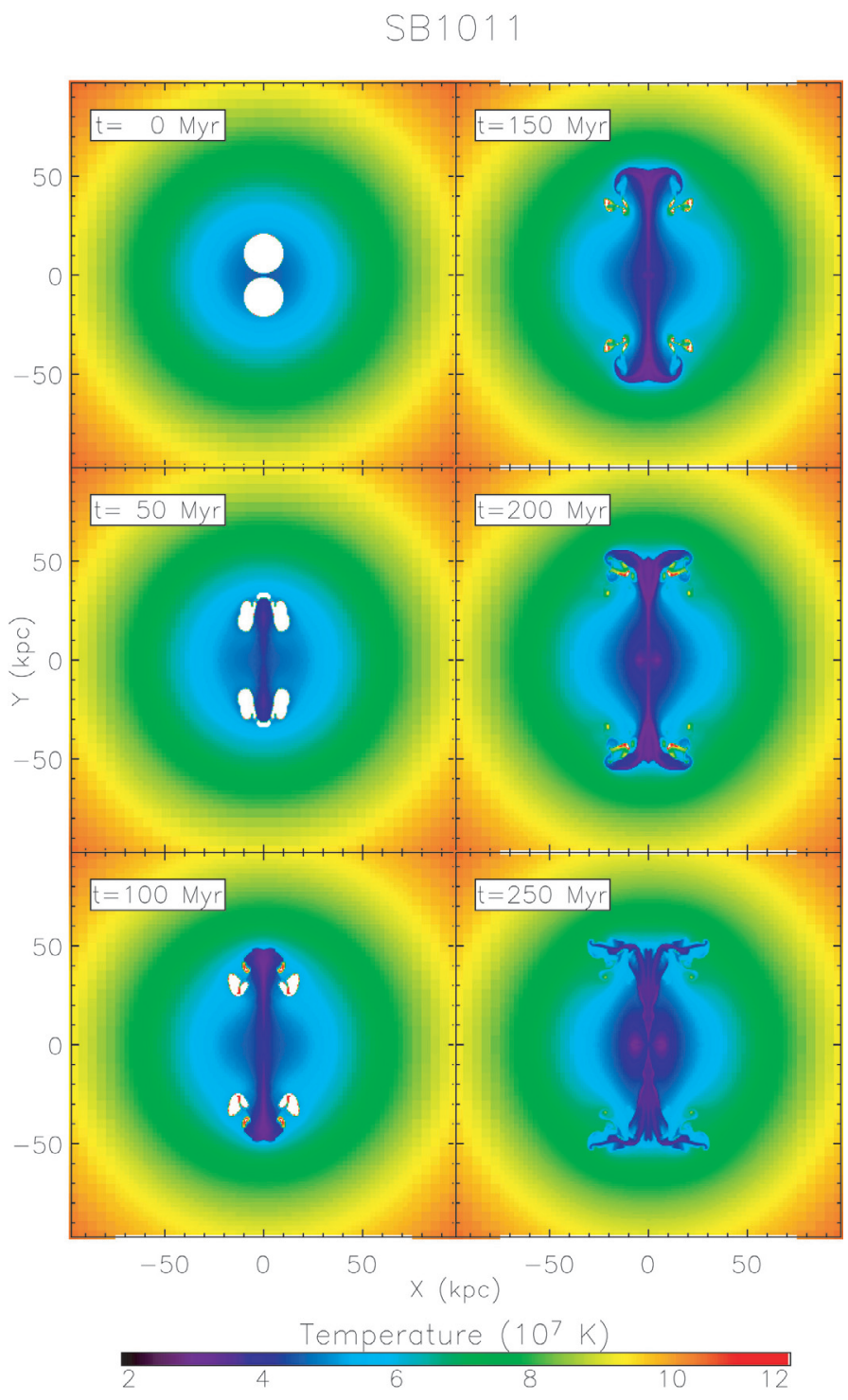

Fig. 6. The evolution of the ICM temperature in the SB1011 simulation from the beginning of the simulation up to $t=250 \mathrm{Myr}$. The bubble plasma seems almost completely diffused into the ICM in last snapshot, but it is still preserved in remnants of the vortex ring that do not intersect the plane of the image. The pictures show the entrainment of cold gas along the vertical axis, the establishment of a cold cap at large radii, the fall back of the cold gas and the development of the cooling flow.

no longer be treated as relativistic. In the vortex ring, the plasma is also mixed with the ICM, but because of the lower density of the polluting ICM, the mixture still preserves buoyancy and a high temperature.

As stated above, despite its relevance for the evolution of the bubbles and the ICM dynamics, mixing is not properly treated in these simulations because the diffusion of species is not explicitly accounted for. We address this point by checking that the amount of mixing remains stable with respect to increases in resolution. We ran a $2 \mathrm{D}$ simulation with the same characteristics as the SB1020 case and compared it with an identical 2D simulation with two additional levels of refinement. We found that both simulations show the same amount of mixing up to $t=250 \mathrm{Myr}$, despite the fact that the plasma distribution shows narrower features in the second case, as expected. We consider that the mixing should eventually increase in the 2D simulations due to their enhanced sensitivity to instabilities; hence the fact

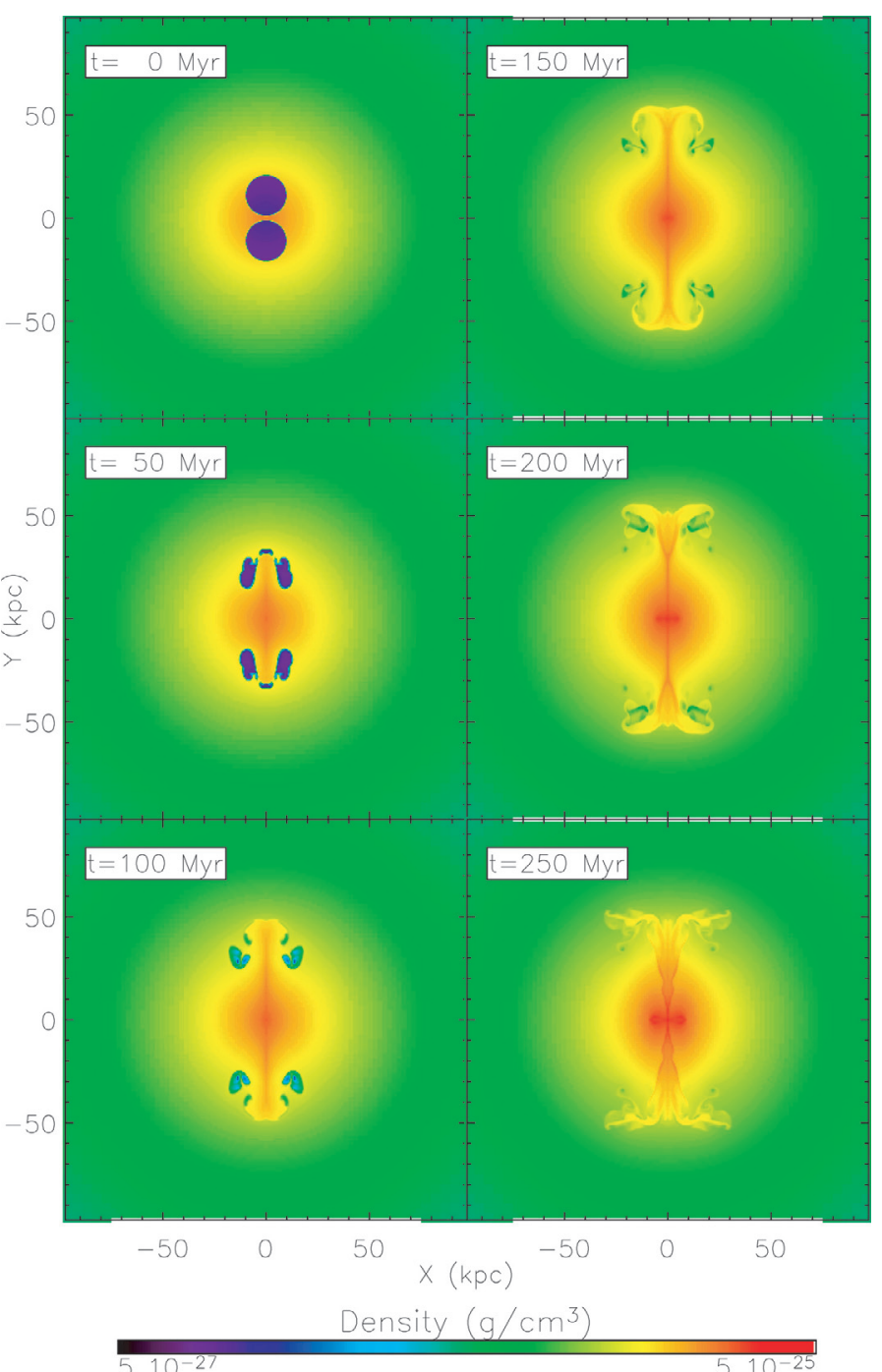

Fig. 7. The evolution of the density in the SB1011 simulation up to $t=$ 250 Myr. The snapshots show the denser material that is uplifted by the vortex circulation and later sustained at large radii by the buoyancy of the vortex ring.

that the amount of mixing remains the same is a proof of its robustness.

The vortex rings evolve similarly in the different simulations. In all of the models the rings are affected by instabilities at regular distances along their length that act to break the rings into smaller sections. It is worth noting that the positions of the break points along the rings are symmetric with respect to the principal vertical planes of the system, and hence the instabilities do not reflect the axial symmetry of the cluster but the geometry of the computational grid. In the SB1011 simulation, the rings are already broken in the last outputs exactly along the plane of the maps, so that it seems in Fig. 6 that only some remnants are slightly hotter than the surrounding medium after $t=200 \mathrm{Myr}$. However, this is not the case: the hot material is concentrated outside of the plane of the figure. In the SB1020 and SB1030 simulations, the vortex rings are not yet broken by the end of the run, but instabilities are effectively breaking them outside the plane of the image. In all of the simulations the rings preserve high temperatures despite the fact that the fraction of bubble plasma inside them is less than $10 \%$. 
SB1011

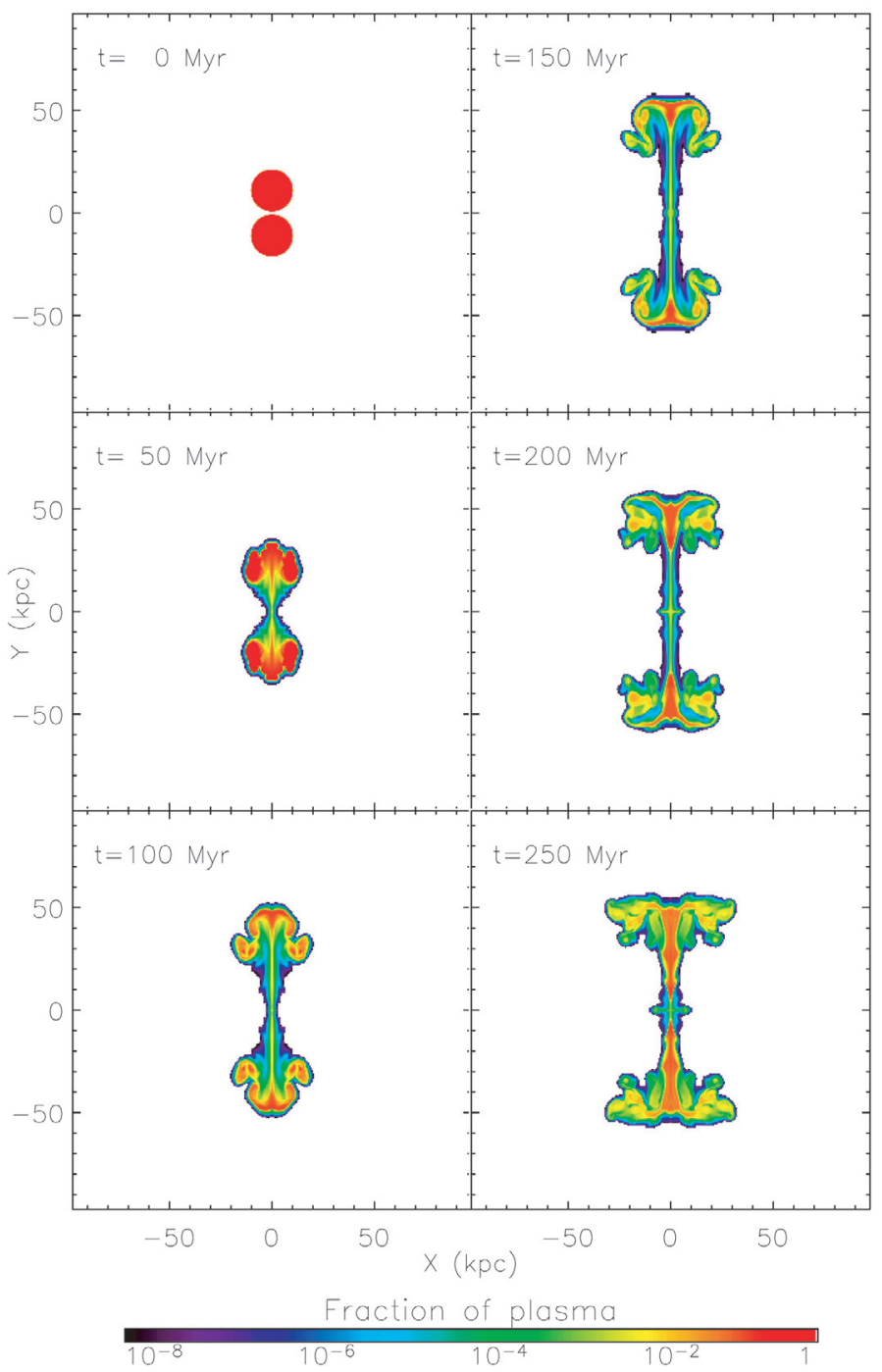

Fig. 8. The fraction of bubble plasma in the fluid elements in the SB1011 simulation up to $t=250$ Myr. The comparison with Figs. 6 and 7 shows that as the simulation goes on, most of the bubble plasma is indeed mixed with the ICM to low temperature and high density, and under these conditions it should no longer be treated as relativistic. The reader should be careful of the logarithmic color scale.

We quantify the evolution of the bubbles in Fig. 10, which shows the height of the centroid of the plasma material as a function of time. The the value of the height of the centroid of all of the plasma is shown, while the same quantity for the plasma that is hotter than $T=8 \times 10^{7} \mathrm{~K}$ (the maximum temperature of the ICM inside $r=80 \mathrm{kpc}$ ) is depicted differently. The thickness of the lines distinguishes among the different simulations. The three simulations show remarkably similar behavior: the hotter material, which effectively defines the bubbles, rises by $\sim 20 \mathrm{kpc}$ in the first $100 \mathrm{Myr}$, and then decreases its speed until the end of the simulation. The average speed is then $\sim 200 \mathrm{~km} \mathrm{~s}^{-1}$ for the first $100 \mathrm{Myr}$, which can be compared to the speed of sound in the ICM, ranging from $\sim 1000 \mathrm{~km} \mathrm{~s}^{-1}$ at the center to $\sim 1500 \mathrm{~km} \mathrm{~s}^{-1}$ at $r=100 \mathrm{kpc}$. The differences between the values for the overall plasma and the hottest plasma at the beginning of the simulation are explained by the fact that, due to the finite grid size, a portion of bubble material is already mixed with the ICM in the initial conditions. Initially this

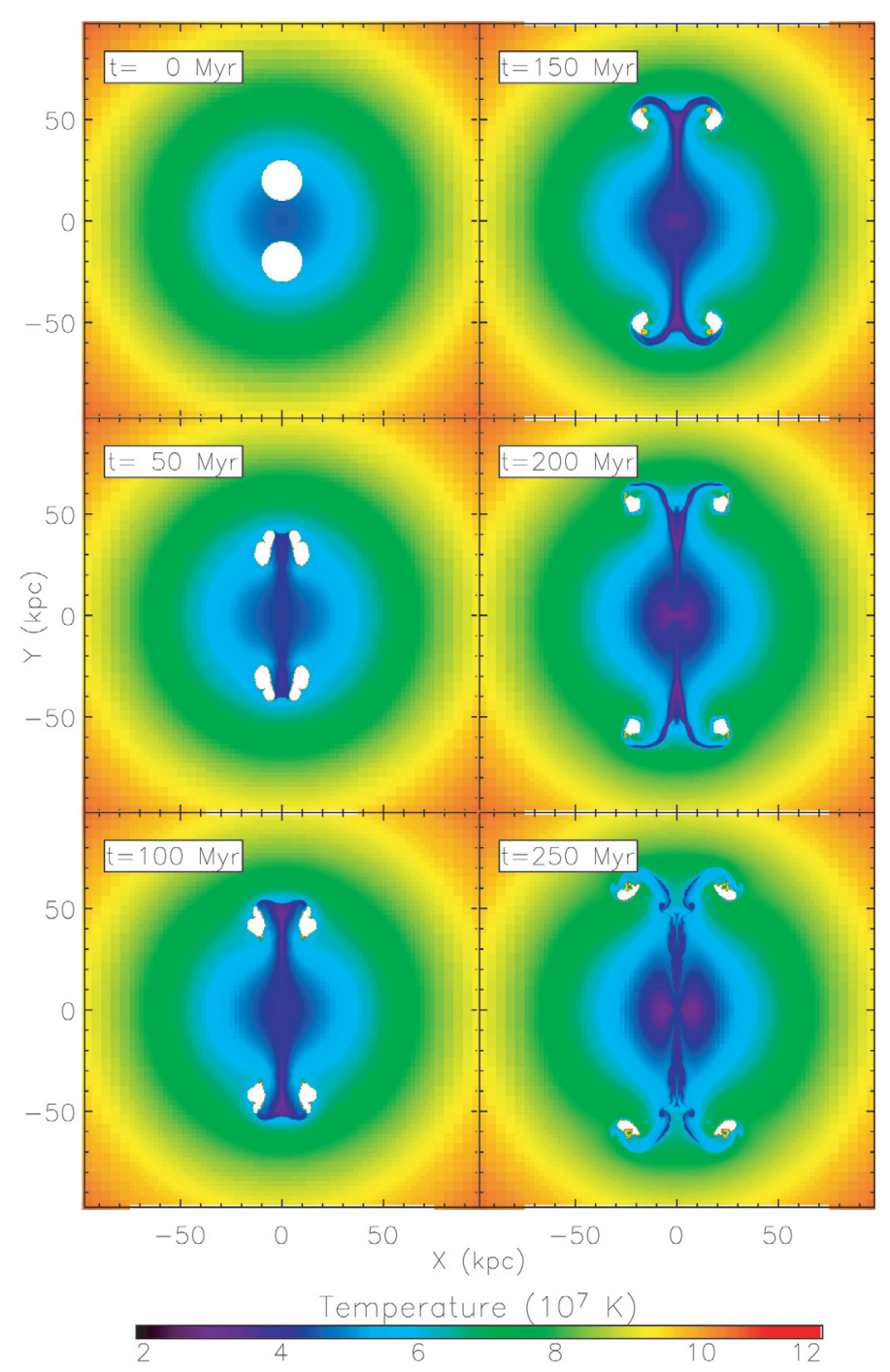

Fig. 9. The evolution of the temperature in the SB1020 simulation.

material is less affected by buoyancy, and it is driven upward later in the bubble wake. The rising of the trunk of cold gas, the sweeping of the plasma on the bubble axis, and the establishment of a cold cap above the ring are also well represented by the rise of the overall centroid higher than the hot plasma. The succeeding decrease depicts the falling back of the cold material along the trunk. The centroid of the hot material still seems to rise steadily in the last outputs of our simulations, but inspection of the fraction of plasma that is still hot, depicted in Fig. 11, shows that the plasma in the SB1011 simulation is rapidly cooling. In the SB1020 and SB1030 simulations, it instead appears that the fraction of hot plasma is stable around $35 \%$ in the final output.

To explore the later stages of the evolution, we turn again to $2 \mathrm{D}$ simulations with the same parameters and resolution as the $3 \mathrm{D}$ cases. In general, these simulations show that material continues to fall along the trunks down to the cluster center, while the ICM already present there is displaced orthogonally and settles into a torus. The trend then reverses and the material in the torus falls back onto the core, driving the material that has fallen along the trunks back upward. Overall, this resembles a sort of oscillatory motion, but it becomes more turbulent as the simulation proceeds. We halt the simulations when some plasma 


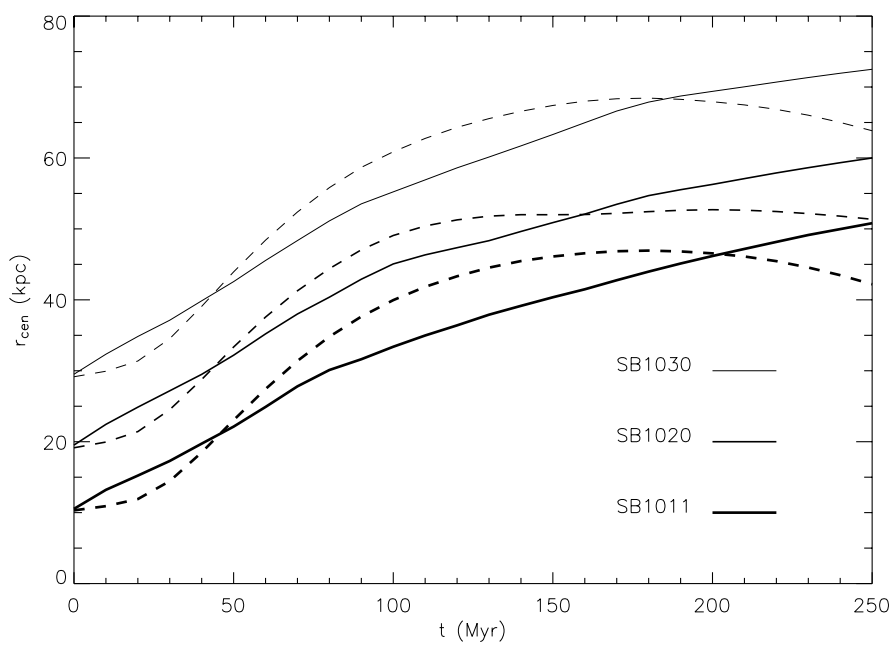

Fig. 10. The height of the centroid of the plasma material during each run for the complete set of bubble simulations. The dashed lines describe the height of the centroid of all the bubble plasma, while the solid lines depict the same quantity for the plasma that is hotter than $T=8 \times 10^{7} \mathrm{~K}$, which is the maximum temperature inside $r=80 \mathrm{kpc}$.

element cools close to the minimum temperature and the computational time step becomes too short; we can suppose that a runaway cooling similar to the homogeneus cooling flow is established at that time. Vortex rings are preserved until the end of the run, deforming and slowly rising for a few more kpc.

\section{Effects of bubbles on the cooling}

It has been observed that AGNs might heat the ICM and possibly quench cooling flows in any of several different ways. In particular, it has been predicted (e.g., Heinz et al. 1998) and shown by simulations (Zanni et al. 2005; Brüggen et al. 2005) that most of the jets' energy is transferred to the ICM through the bow shock and the sound waves pushed by the expanding cocoon. These processes deposit energy into the ICM as the jets cross it, affecting the ICM at a distance from the radio source itself and possibly outside the cool core, unless some viscosity or thermal conductivity is present (Ruszkowski et al. 2004a,b; Brüggen et al. 2005; Fabian et al. 2005). On the other hand, energy is still stored in the cavities as thermal energy and in the ICM in potential form during the later phase of evolution by buoyancy. Both these forms of energy are released during the rise of the cavities: the first through $p d V$ work performed by the cavities, which expand to reach or maintain pressure equilibrium with the environment (e.g., Begelman 2001), and the second when the surrounding medium falls in around the cavity to fill the space the bubble previously occupied (e.g., Churazov et al. 2002; Bîrzan et al. 2004). Work and thermal energy exchange also occur when portions of the ICM at different temperatures and pressures come into contact and mix, after being displaced by motions induced by the rise of the cavities (Quilis et al. 2001; Churazov et al. 2002; Dalla Vecchia et al. 2004). This mechanism becomes more effective in heating the cool core as more material is radially displaced.

Despite the presence of bubbles, our simulations show that a cooling flow is established in the central regions of the cluster (Figs. 6 and 9). This is even more true as the initial location of the bubbles is moved away from the center. This is hardly surprising, given that bubbles are the outcome of a single burst of energy injection while cooling is continously operating in the

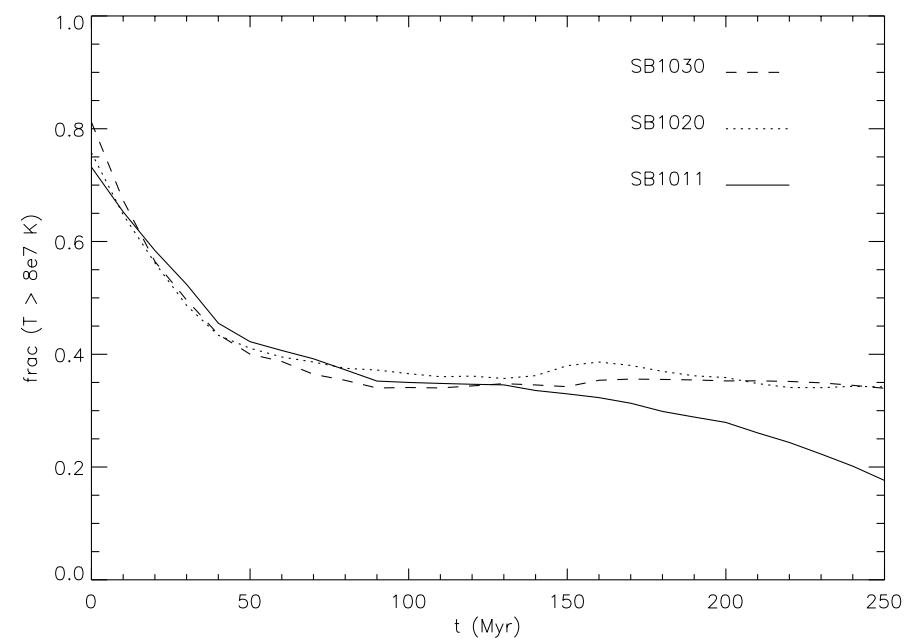

Fig. 11. The fraction of bubble plasma that maintains a temperature $T>$ $8 \times 10^{7} \mathrm{~K}$ as a function of time. Even if the temperature of this plasma is lowered to the non-relativistic regime, it is still hot and underdense enough to be driven by buoyancy.

ICM. Nonetheless, bubbles affect the ICM and can slow down its cooling effectively; in the following we investigate how and by what amount.

Because of the dependence of gas emissivity upon density (approximately $\epsilon \propto \rho^{2} T^{-0.6}$ ), cooling is enhanced by deposition and compression of material. Thus, we observe that bubbles affect the cooling firstly by their mere presence, because of the removal from their volumes of some fraction of the cold ICM already stored in the core. Even if this can be regarded as an artifact of our setup, we expect that a similar effect is produced on the ICM by displacement and shock heating during bubble inflation. Later, some amount of thermal energy is directly deposited in the ICM through mixing with the bubble material, while stirring of the ICM due to the bubble rise is expected to reduce the deposition of cooling material to the core. To investigate the effects of the gas motions on the cooling, we performed a new run of the SB1020 case using a tracer fluid to mark the fluid elements initially stored inside $r=10 \mathrm{kpc}$; we show in Fig. 12 the evolution of their distribution. We observe that in the first stages of evolution, the central material is stretched and uplifted in the bubbles' wake and expands rather than being compressed. Later on, material indeed falls onto the core, but because of the geometry, it accumulates directly at the center rather than on the cold ICM, which is instead displaced into a torus. A similar analysis was also performed in the SB1011 case; its results are similar to SB1020 up to $t=190 \mathrm{Myr}$, when the run halts.

We measure the effects of the bubbles on the cooling by comparing the temperature profiles with the profiles of the $\mathrm{CO} 2$ model. This is done in Fig. 13, which reports the difference $T-T_{\mathrm{CO} 2}$ from the initial conditions up to $t=250 \mathrm{Myr}$ with intervals of 50 Myr. It must be remembered that, as depicted in Fig. 3, in the absence of bubbles the gas cools about $\Delta T \sim 2 \times 10^{7} \mathrm{~K}$ during the first $250 \mathrm{Myr}$ of evolution, while the cooling is much faster at later times. Hence, a slight gain in the core temperature can be indicative of a significantly longer evolution of the cooling process. The bubbles in simulation SB1011 have the greatest effect on the cooling, as expected, due to their proximity to the core of the cluster. In this case the temperature is increased with respect to the $\mathrm{CO} 2$ simulation in all of the regions inside $r \sim 50 \mathrm{kpc}$ for most of the run. At $t=250 \mathrm{Myr}$, the temperature difference reaches $T-T_{\mathrm{CO} 2} \sim 10^{7} \mathrm{~K}$. The most interesting 


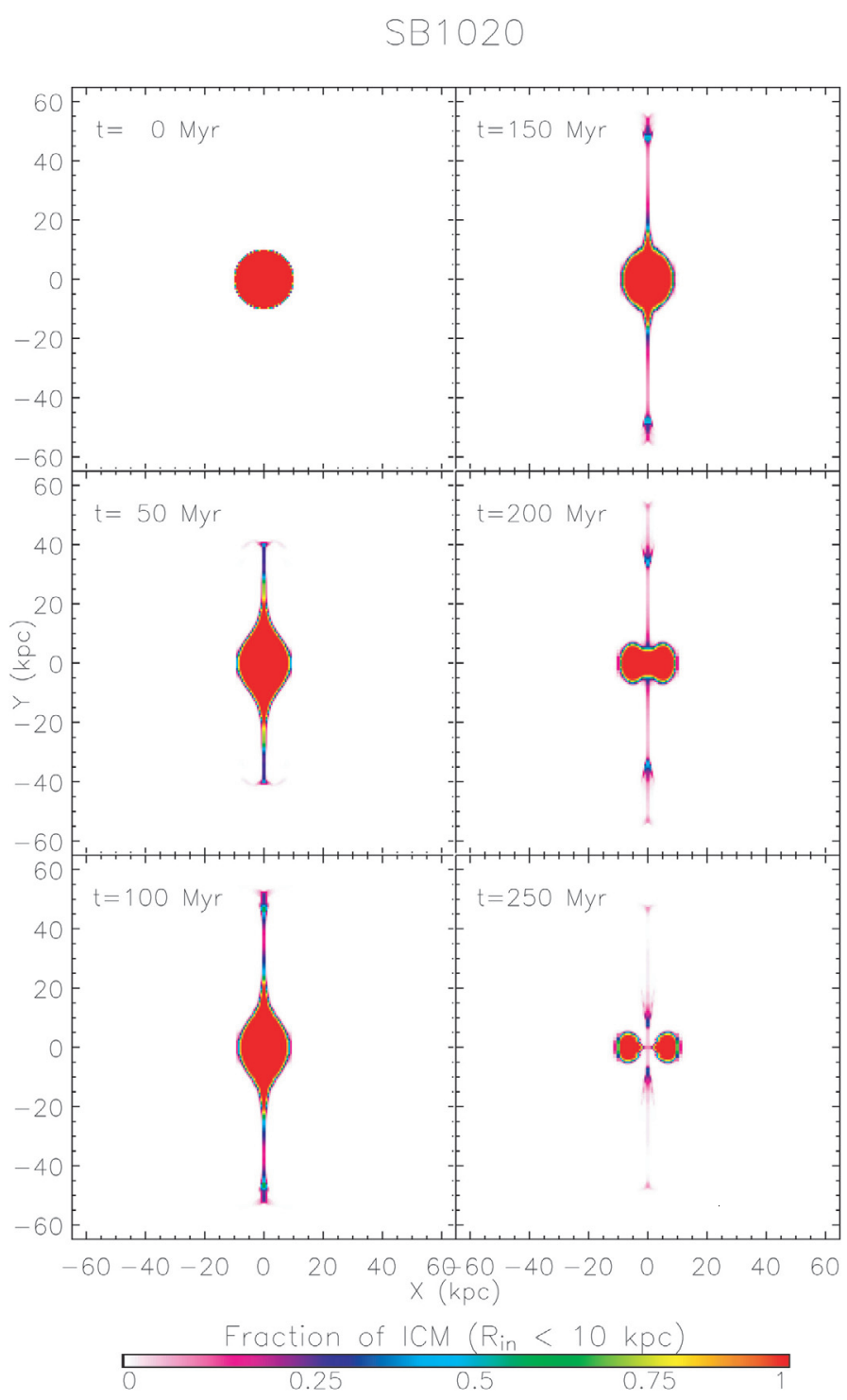

Fig. 12. The evolution of the material initially inside $r=10 \mathrm{kpc}$ in run SB1020. It is first stretched by the rise of the bubbles and later displaced into a torus around the center by the material falling along the trunks.

result comes from the SB1020 simulation. In this case the cooling in the center proceeds apparently as in the $\mathrm{CO} 2$ case up to $t \sim 200$ Myr. After this time, however, the central temperature drops more slowly, and in the final snapshot $T-T_{\mathrm{CO} 2} \sim 6 \times 10^{6} \mathrm{~K}$. This is due to the replacement of the cold material in the core by the dense gas falling to the center along the trunks. In the SB1030 simulation, the core of the cluster instead appears completely unaffected by the presence of bubbles up to the end of the run at $t=250 \mathrm{Myr}$. However, because dense gas is clearly falling along the trunks at that time, a later heating as in SB1020 cannot be excluded. Because of the fraction of plasma entrained in the core (Fig. 8), we wondered if the higher temperature of the infalling material was due to mixing. Therefore we computed the profiles of the fraction of internal energy due to the bubble plasma as shown in Fig. 14. At $t=250 \mathrm{Myr}$, we found that the internal energy directly deposited in the ICM amounts at most at $2 \%$ everywhere except in the shells hosting the vortex rings.

The use of 2D simulations enables us to extend this analysis up to the establishment of runaway cooling when some gas element reaches the mimimum temperature. In the case of the

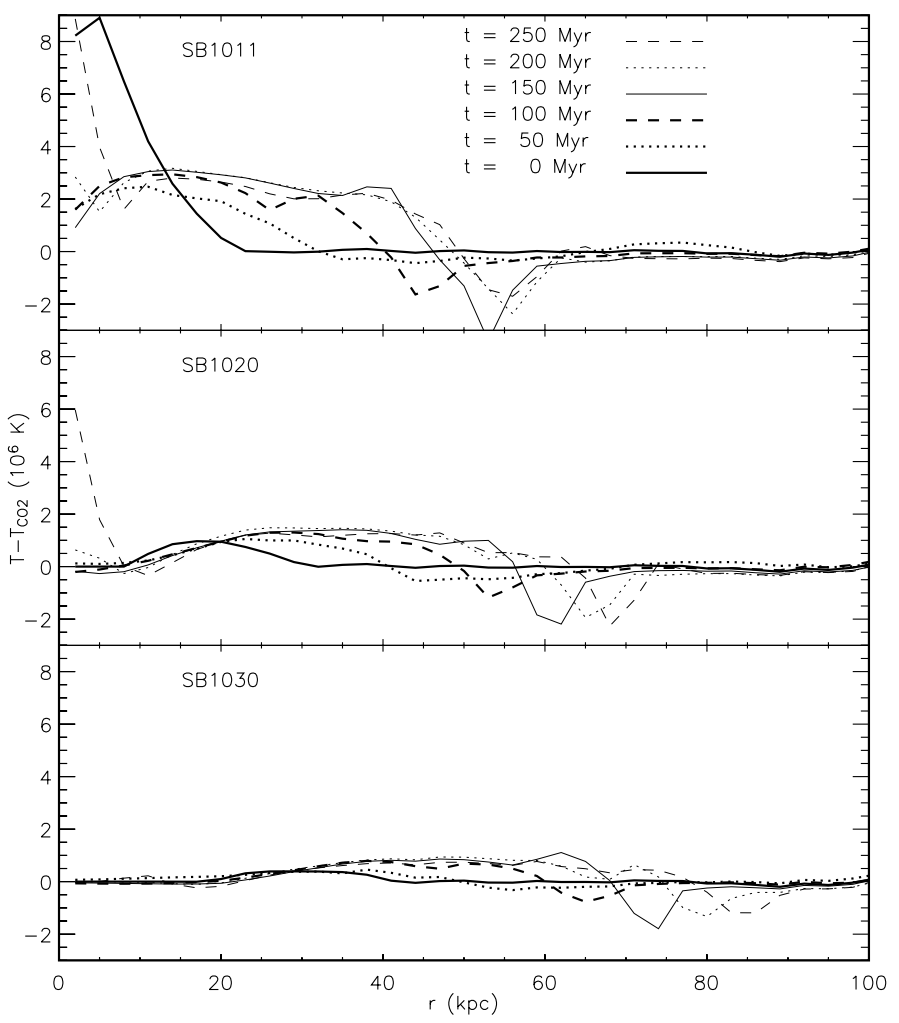

Fig. 13. The difference between the temperature profile of the simulations hosting bubbles and the $\mathrm{CO} 2$ case.

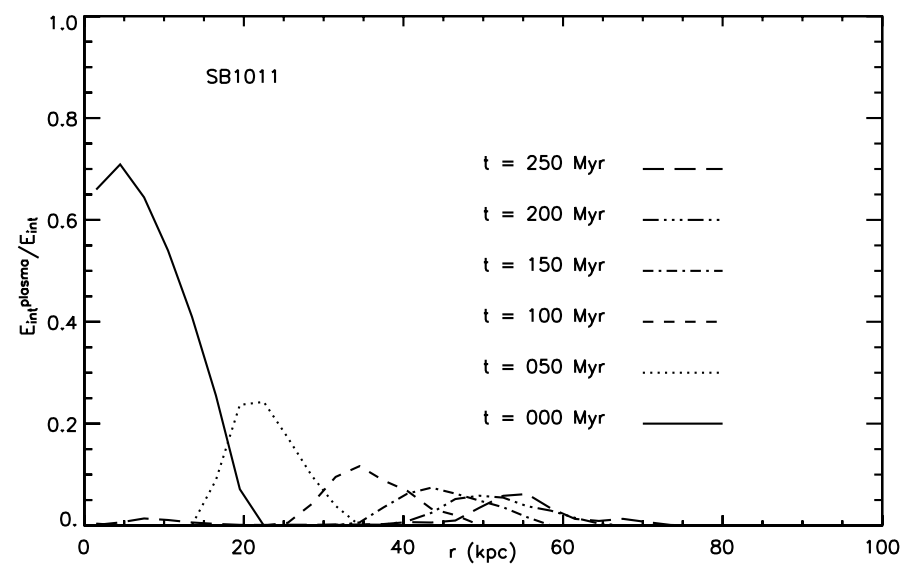

Fig. 14. Profiles of the fraction of the internal energy due to the bubble plasma. The ratio is computed assuming that the fraction of plasma in each radial shell still hosts the same specific energy as in the initial conditions.

homogeneus cooling flow, we saw that this happens soon after $t=390$ Myr. In SB1011, after a turbulent oscillation, runaway cooling is established in the core at some time after $t=500 \mathrm{Myr}$. SB1020 presents an analogous behavior, but because of the later infall, the cooling flow is more rapidly re-established and the runaway phase starts at $t=440 \mathrm{Myr}$. In the SB1030 case, the infalling material does not reach the center, but rather compresses the material already present in the core; the effect is to enhance the cooling so that the runaway phase begins after $t=380 \mathrm{Myr}$.

\section{Conclusions}

We show the results of a set of 3D simulations of buoyant bubbles in the atmosphere of a cool core cluster. We allow cooling, 
consider the bubble plasma as relativistic, and achieve high effective resolution. We consider the presented simulations to be a first step in studying the interaction of plasma bubbles with the ICM. We will use these runs as references for future work with different configurations of the system and additional physics.

The dynamics of our bubbles resembles the results described by Saxton et al. (2001) and by Reynolds et al. (2005). Saxton et al. (2001) produce a set of 2D simulations similar to SB1030 for different values of the ratio of the density of plasma versus ICM. They remark upon the robustness of the vortex rings and the fall of entrained material to the core along the trunks; as in SB1030, this material simply deposits onto the core rather than disrupts it. Reynolds et al. (2005) study the evolution of bubbles for different values of the ICM viscosity using 3D simulations. In a reference case without viscosity, the bubble evolves similarly to SB1011, deforming in vortex rings with a thin trunk of material entrained in its wake. Each of these studies focuses on the evolution of bubbles, rather than on their impact on the environment, and therefore neglects cooling.

Our simulations do not fully address the problem of the quenching of the cooling flow by AGN because the phase of activity is neglected and many simplifications are assumed in the setup. We address, however, the direct effect of our bubbles on the cooling and find that it is rather modest. Even in the SB1011 case, runaway cooling is postponed only by about $25 \%$ with respect to the unperturbed case. Setting the bubbles further away only reduces their effect, or even accelerates the cooling as in SB1030. Larger bubbles will probably have a bigger impact, but the dimensions chosen in our cases are typical (Bîrzan et al. 2004).

Numerically, the results are quite robust with respect to increase in resolution and the adoption of different systems of coordinates. Thus we conclude that our resolution is adequate. Details are different, however, and 2D simulations are much more sensitive to the propagation of rounding errors. For this reason we prefer to trust the $3 \mathrm{D}$ cases and use $2 \mathrm{D}$ simulations only to extend their results. To a lesser extent, 3D simulations suffer numerical instabilities as well, as revealed by the shredding of vortex rings along the lines of the computational grid. One technique for mitigating grid effects involves just adding some random noise in the initial conditions, as in Jones \& De Young (2005). The symmetry of our results, however, testifies to the reliability of the code.

Future work will consider some relevant physics that is not included in the present simulations. In particular, we will consider the magnetic field and the ICM viscosity, which have been shown to be able, for proper values, to stabilize the bubble surface. Using more realistic initial conditions, we will take into better account the activity phase of AGN and its effects on the medium. The introduction of thermal conduction can be relevant in heating the entrained cold gas as it rises to regions of higher temperature, besides directly heating the cluster core. Mixing of hot and cold gas should also be properly treated, as well as the transition of the plasma to the non-relativistic regime. We also aim to study the interaction of pre-existing bubbles with shock waves induced by AGN activity or merger events (Enßlin \& Brüggen 2002; Heinz \& Churazov 2005). Finally, a direct comparison with X-ray observations is possible using tools like X-MAS (Gardini et al. 2004).

Acknowledgements. The author acknowledges support under a Presidential Early Career Award from the US Department of Energy, Lawrence Livermore National Laboratory (contract B532720), as well as the National Center for Supercomputing Applications. He wishes to thank Paul Ricker for his huge contribution to many aspects of this work. He also wishes to thank Robert
Wilhelmson for useful reading suggestions. FLASH was developed at the University of Chicago ASC Flash Center under support provided by Department of Energy grant number B341495.

\section{References}

Alexander, P. 2002, MNRAS, 335, 610

Allen, S. W., Ettori, S., \& Fabian, A. C. 2001, MNRAS, 324, 877

Ball, R., Burns, J. O., \& Loken, C. 1993, AJ, 105, 53

Bałucińska-Church, M., Ostrowski, M., Stawarz, Ł., \& Church, M. J. 2005, MNRAS, 357, L6

Barthel, P. D., \& Arnaud, K. A. 1996, MNRAS, 283, L45

Basson, J. F., \& Alexander, P. 2003, MNRAS, 339, 353

Batchelor, G. K. 1967, An Introduction to Fluid Dynamics (Cambridge: Cambridge Univ. Press)

Begelman, M. C. 2001, Impact of Active Galactic Nuclei on the Surrounding Medium in Gas and Galaxy Evolution, ed. J. E. Hibbard, M. Rupen, \& J. H. van Gorkom. San Francisco: Astronomical Society of the Pacific, ASP Conf. Proc. 240, 363

Begelman, M. C., \& Cioffi, D. F. 1989, ApJ, 345, L21

Bîrzan, L., Rafferty, D. A., McNamara, B. R., Wise, M. W., \& Nulsen, P. E. J. 2004, ApJ, 607, 800

Blandford, R. D., \& Rees, M. J. 1974, MNRAS, 169, 395

Blanton, E. L., Sarazin, C. L., McNamara, B. R., \& Wise, M. W. 2001, ApJ, 558, L15

Böhringer, H., Matsushita, K., Churazov, E., Ikebe, Y., \& Chen, Y. 2002, A\&A, 382,804

Brighenti, F., \& Mathews, W. G. 2002a, ApJ, 573, 542

Brighenti, F., \& Mathews, W. G. 2002b, ApJ, 574, L11

Brighenti, F., \& Mathews, W. G. 2003, ApJ, 587, 580

Brüggen, M. 2003a, ApJ, 593, 700

Brüggen, M. 2003b, ApJ, 592, 893

Brüggen, M., \& Kaiser, C. R. 2001, MNRAS, 325, 676

Brüggen, M., \& Kaiser, C. R. 2002, Nature, 418, 301

Brüggen, M., Kaiser, C. R., Churazov, E., \& Enßlin, T. A. 2002, MNRAS, 331, 545

Brüggen, M., Ruszkowski, M., \& Hallman, E. 2005, ApJ, 630, 740

Burns, J. O. 1990, AJ, 99, 14

Churazov, E., Forman, W., Jones, C., \& Böhringer, H. 2000, ApJ, 356, 788

Churazov, E., Brüggen, M., Kaiser, C. R., Böhringer, H., \& Forman, W. 2001, ApJ, 554, 261

Churazov, E., Yaev, R., Forman, W., \& Böhringer, H. 2002, MNRAS, 332, 729

Clarke, D. A., Harris, D. E., \& Carilli, C. L. 1997, MNRAS, 284, 981

Colella, P., \& Woodward, P. 1984, JCP, 54, 174

Colella, P., \& Glaz, H. M. 1985, JCP, 59, 264

David, L. P., Nulsen, P. E. J., McNamara, B. R., et al. 2001, ApJ, 557, 546

Dalla Vecchia, C., Bower, R. G., Theuns, T., et al. 2004, MNRAS, 355, 995

De Grandi, S., \& Molendi, S. 2002, ApJ, 567, 163

Eilek, J. A. 2004, Proceedings of the conference X-Ray and Radio Connections, ed. L. O. Sjowerman, \& K. K. Dyer

Enßlin, T. A., \& Brüggen, M. 2002, MNRAS, 331, 1011

Fabian, A. C. 1994, ARA\&A, 32, 277

Fabian, A. C., Sanders, J. S., Ettori, S., et al. 2000, MNRAS, 318, L65

Fabian, A. C., Sanders, J. S., Allen, S. W., et al. 2003a, MNRAS, 344, L43

Fabian, A. C., Sanders, J. S., Crawford, C. S., et al. 2003b, MNRAS, 344, L48

Fabian, A. C., Reynolds, C. S., Taylor, G. B., \& Dunn, R. J. H. 2005, MNRAS, 363,891

Fabian, A. C., Sanders, J. S., Taylor, G. B., et al. 2006, MNRAS, 366, 417

Forman, W., Nulsen, P., Heinz, S., et al. 2005, ApJ, 635, 894

Fryxell, B., Olson, K., Ricker, P. et al. 2000, ApJS, 131, 273

Gardini, A., \& Ricker, P. M. 2004, MPLA, 19, 2317

Gardini, A., Rasia, E., Mazzotta, P., et al. 2004, MNRAS, 351, 505

Gull, S. F., \& Northover, K. J. E. 1973, Nature, 244, 80

Heinz, S., \& Churazov, E. 2005, ApJ, 634, L141

Heinz, S., Reynolds, C. S., \& Begelman, M. C. 1998, ApJ, 501, 126

Heinz, S., Choi, Y., Reynolds, C. S., \& Begelman, M. C. 2002, ApJ, 569, L79

Hill, M. J. M. 1894, Phil. Trans. Roy. Soc. A, 185

Jetha, N. N., Sakelliou, I., Hardcastle, M. J., Ponman, T. J., \& Stevens, I. R. 2005, MNRAS, 358, 1394

Jones, T. W., \& De Young, D. S. 2005, ApJ, 624, 586

Kaastra, J. S., Tamura, T., Peterson, J. R., et al. 2004, A\&A, 413, 415

Kaiser, C. R., \& Binney, J. 2003, MNRAS, 338, 837

Kaiser, C. R., Pavlovski, G., Pope, E. C. D., \& Fangohr, H. 2005, MNRAS, 359 , 493

Kim, W.-T., \& Narayan, R. 2003, ApJ, 596, L139

Kraft, R. P., Vázquez, S. E., Forman, W. R., et al. 2003, ApJ, 2003, 592, 129

Krause, M. 2003, A\&A, 398, 113 
Krause, M., \& Camenzind, M. 2003, in Transactions of the HLRS, Springer Lecture Notes, ed. E. Krause, W. Jager, \& M. Resch

Löhner, R. 1987, Comp. Meth. Appl. Mech. Eng., 61, 323

Makishima, K., Ezawa, H., Fukuzawa, Y., et al. 2001, PASJ, 53, 401

Markevitch, M., Ponman, T. J., Nulsen, P. E. J., et al. 2000, ApJ, 541, 542

Markevitch, M., Vikhlinin, A., \& Mazzotta, P. 2001, ApJ, 562, L153

Mathews, W. G., Brighenti, F., Buote, D. A., \& Lewis, A. D. 2003, ApJ, 596, 159

Mathews, W. G., Brighenti, F., \& Buote, D. A. 2004, ApJ, 615, 662

Mathews, W. G., Faltenbacher, A., \& Brighenti, F. 2006, ApJ, 638, 659

Mazzotta, P., Markevitch, M., Vikhlinin, A., et al. 2001a, ApJ, 555, 205

Mazzotta, P., Markevitch, M., Forman, W. R., et al. 2001b, ApJ, submitted [arXiv: astro-ph/0108476]

Mazzotta, P., Edge, A. C., \& Markevitch, M. 2003, ApJ, 596, 190

McNamara, B. R., Wise, M. W., Nulsen, P. E. J., et al. 2001, ApJ, 562, L149

McNamara, B. R., Nulsen, P. E. J., Wise, M. W., et al. 2005, Nature, 433, 45

Narayan, R., \& Medvedev, M. K. 2001, ApJ, 562, L129

Navarro, J. F., Frenk, C. S., \& White, S. D. M. 1997, ApJ, 490, 493 (NFW)

Nulsen, P. E. J., Hambrick, D. C., McNamara, B. R., et al. 2005a, ApJ, 625, L9

Nulsen, P. E. J., McNamara, B. R., Wise, M. W., \& David, L. P. 2005b, ApJ, 636, 628

Omma, H., \& Binney, J. 2004, MNRAS, 350, L13

Omma, H., Binney, J., Bryan, G., \& Slyz, A. 2004, MNRAS, 348, 1105

Peterson, J. R., Paerels, F. B. S., Kaastra, J. S., et al. 2001, A\&A, 365, L104

Peterson, J. R., Kahn, S. M., Paerels, F. B., et al. 2003, ApJ, 590, 207

Quilis, V., Bower, R. G., \& Balogh, M. L. 2001, MNRAS, 328, 1091

Reynolds, C. S., Heinz, S., \& Begelman, M. C. 2001, ApJ, 549, L179

Reynolds, C. S., Heinz, S., \& Begelman, M. C. 2002, MNRAS, 332, 271

Reynolds, C. S., McKernan, B., Fabian, A. C., Stone, J. M., \& Vernaleo, J. C. 2005, MNRAS, 357, 242
Rizza, E., Loken, C., Bliton, M., et al. 2000, AJ, 119, 21

Robinson, K., Dursi, L. J., Ricker, P. M., et al. 2004, ApJ, 601, 621

Roychowdhury, S., Ruszkowski, M., Nath, B. B., \& Begelman, M. C. 2004, ApJ, 615,681

Ruszkowski, M., \& Begelman, M. C. 2002, ApJ, 581, 223

Ruszkowski, M., Brüggen, M., \& Begelman, M. C. 2004a, ApJ, 611, 158

Ruszkowski, M., Brüggen, M., \& Begelman, M. C. 2004b, ApJ, 615, 675

Sanderson, A. J. R., Finoguenov, A., \& Mohr, J. J. 2005, ApJ, 630, 191

Sarazin, C. L. 1988, X-ray Emission from Clusters of Galaxies (Cambridge: Cambridge Univ. Press)

Saxton, C. J., Sutherland, R. S., \& Bicknell, G. V. 2001, ApJ, 563, 103

Scheuer, P. A. G. 1974, MNRAS, 166, 513

Smith, M. D., Smarr, L., Norman, M. L., \& Wilson, J. R. 1983, ApJ, 264, 432

Smith, D. A., Wilson, A. S., Arnaud, K. A., Terashima, Y., \& Young, A. J. 2002, ApJ, 565, 195

Soker, N., \& Sarazin, C. L. 1990, ApJ, 348, 73

Soker, N., Blanton, E. L., \& Sarazin, C. L. 2002, ApJ, 573, 533

Spergel, D. N., Verde, L., Peiris, H. V., et al. 2003, ApJS, 148, 175

Sutherland, R. S., \& Dopita, M. A. 1993, ApJS, 88, 253

Tamura, T., Kaastra, J. S., Peterson, J. R., et al. 2001, A\&A, 365, L87

Turner, J. S. 1973, Buoyancy Effects in Fluids (Cambridge: Cambridge Univ. Press)

Vernaleo, J. C., \& Reynolds, C. S. 2006, ApJ in press [arXiv: astro-ph/0511501]

Voigt, L. M., \& Fabian, A. C. 2004, MNRAS, 347, 1130

Walters, J. K., \& Davidson, J. F. 1963, J. Fluid Mech., 17, 321

White, D. A., \& Sarazin, D. L. 1987, ApJ, 320, 32

Zakamska, N. L., \& Narayan, R. 2003, ApJ, 582, 162

Zanni, C., Bodo, G., Rossi, P., et al. 2003, A\&A, 402, 949

Zanni, C., Murante, G., Bodo, G., et al. 2005, A\&A, 429, 399 